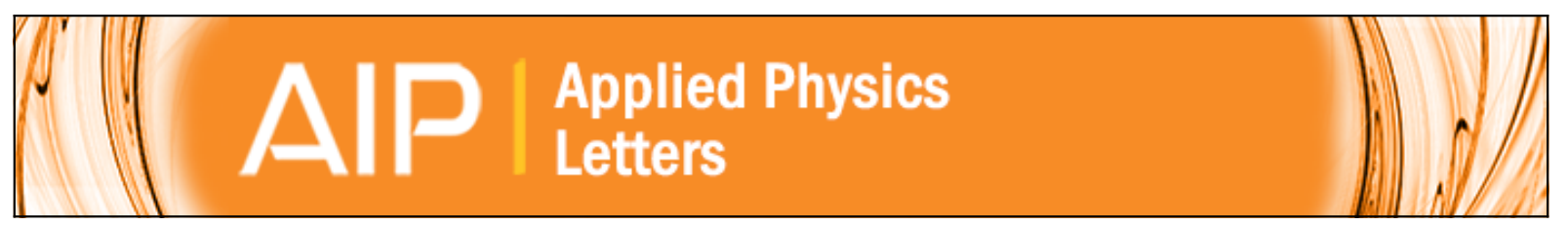

\title{
Electrical instability of amorphous indium-gallium-zinc oxide thin film transistors under monochromatic light illumination
}

Xiaoming Huang, Chenfei Wu, Hai Lu, Fangfang Ren, Qingyu Xu, Huiling Ou, Rong Zhang, and Youdou Zheng

Citation: Applied Physics Letters 100, 243505 (2012); doi: 10.1063/1.4729478

View online: http://dx.doi.org/10.1063/1.4729478

View Table of Contents: http://scitation.aip.org/content/aip/journal/apl/100/24?ver=pdfcov

Published by the AIP Publishing

\section{Articles you may be interested in}

A thermalization energy analysis of the threshold voltage shift in amorphous indium gallium zinc oxide thin film transistors under simultaneous negative gate bias and illumination

J. Appl. Phys. 115, 134501 (2014); 10.1063/1.4870457

Temperature dependence of negative bias under illumination stress and recovery in amorphous indium gallium zinc oxide thin film transistors

Appl. Phys. Lett. 102, 143506 (2013); 10.1063/1.4801762

Light induced instabilities in amorphous indium-gallium-zinc-oxide thin-film transistors

Appl. Phys. Lett. 97, 173506 (2010); 10.1063/1.3503971

Electrical stress-induced instability of amorphous indium-gallium-zinc oxide thin-film transistors under bipolar ac stress

Appl. Phys. Lett. 95, 132101 (2009); 10.1063/1.3237169

Origin of threshold voltage instability in indium-gallium-zinc oxide thin film transistors

Appl. Phys. Lett. 93, 123508 (2008); 10.1063/1.2990657

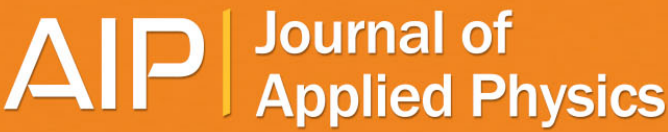

Journal of Applied Physics is pleased to announce André Anders as its new Editor-in-Chief 


\title{
Electrical instability of amorphous indium-gallium-zinc oxide thin film transistors under monochromatic light illumination
}

\author{
Xiaoming Huang, ${ }^{1}$ Chenfei Wu, ${ }^{1}$ Hai Lu, ${ }^{1, a)}$ Fangfang Ren, ${ }^{1}$ Qingyu $\mathrm{Xu},{ }^{2}$ Huiling Ou, ${ }^{2}$ \\ Rong Zhang, ${ }^{1}$ and Youdou Zheng ${ }^{1}$ \\ ${ }_{1}^{1}$ Jiangsu Provincial Key Laboratory of Advanced Photonic and Electronic Materials, and School of Electronic \\ Science and Engineering, Nanjing University, Nanjing 210093, China \\ ${ }^{2}$ Department of Physics, Southeast University, Nanjing 211189, China
}

(Received 14 March 2012; accepted 31 May 2012; published online 14 June 2012)

\begin{abstract}
The electrical instability behaviors of a positive-gate-bias-stressed amorphous indium-gallium-zinc oxide (a-IGZO) thin film transistor (TFT) are studied under monochromatic light illumination. It is found that as the wavelength of incident light reduces from $750 \mathrm{~nm}$ to $450 \mathrm{~nm}$, the threshold voltage of the illuminated TFT shows a continuous negative shift, which is caused by photo-excitation of trapped electrons at the channel/dielectric interface. Meanwhile, an increase of the sub-threshold swing (SS) is observed when the illumination wavelength is below $625 \mathrm{~nm}(\sim 2.0 \mathrm{eV})$. The SS degradation is accompanied by a simultaneous increase of the field effect mobility $\left(\mu_{\mathrm{FE}}\right)$ of the TFT, which then decreases at even shorter wavelength beyond $540 \mathrm{~nm}(\sim 2.3 \mathrm{eV})$. The variation of SS and $\mu_{\mathrm{FE}}$ is explained by a physical model based on generation of singly ionized oxygen vacancies $\left(\mathrm{V}_{\mathrm{o}}^{+}\right)$ and double ionized oxygen vacancies $\left(\mathrm{V}_{\mathrm{o}}{ }^{2+}\right)$ within the a-IGZO active layer by high energy photons, which would form trap states near the mid-gap and the conduction band edge, respectively. (C) 2012 American Institute of Physics. [http://dx.doi.org/10.1063/1.4729478]
\end{abstract}

Transparent oxide-based thin film transistors (TFTs) have been attracting much attention recently due to their excellent electrical and optical characteristics for display applications. In particular, amorphous indium-gallium-zinc oxide (a-IGZO) TFTs are intensively investigated as a replacement for silicon-based TFTs in active matrix displays as they could simultaneously offer high channel electron mobility, high optical transparency, low off-state leakage, and low processing temperature. ${ }^{1,2}$ Although a-IGZO TFTs have shown good performance, these devices still generally suffer from a number of reliability problems. In particular, since switching TFTs used for liquid crystal or organic light emitting diode displays are almost inevitably exposed to light during operation, it is very important to investigate and improve the reliability characteristics of a-IGZO TFTs under various light illumination conditions.

Recently, although several studies have reported that aIGZO TFTs could exhibit electrical instability under ultraviolet or sub-bandgap light illumination, these reports mainly focus on illumination-induced threshold voltage $\left(\mathrm{V}_{\mathrm{th}}\right)$ shift of the TFTs, which is explained as being carrier trapping/ detrapping-related. $^{3-7}$ Comparatively, detailed electrical transport properties of the a-IGZO TFTs under various monochromatic light illumination are less studied. In this report, we find that at certain photon energy range, subthreshold swing (SS) and field effect mobility $\left(\mu_{\mathrm{FE}}\right)$ of the illuminated TFTs could also exhibit instability behavior, which is explained by a transport model based on oxygen-vacancyrelated new trap generation.

The back-gate a-IGZO TFTs studied in this work are fabricated on heavily doped n-type silicon substrate. A $200 \mathrm{~nm} \mathrm{SiO}_{2}$ gate insulator is firstly deposited by plasma

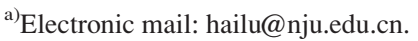

enhanced chemical vapor deposition at $300^{\circ} \mathrm{C}$ on wafer front side, which is followed by deposition of a $50 \mathrm{~nm}$ a-IGZO active layer by using pulsed laser deposition at room temperature in an oxygen partial pressure of $0.5 \mathrm{~Pa}$. The composition of the ceramic target used is $\operatorname{In}_{2} \mathrm{O}_{3}: \mathrm{Ga}_{2} \mathrm{O}_{3}: \mathrm{ZnO}=1: 1: 1$ in mole ratio. The active device region is then defined by optical photolithography and wet chemical etching. Next, the source/drain contact electrodes consisting of Ti/Au (30/ $70 \mathrm{~nm}$ ) bi-layer are deposited by e-beam evaporation and are further patterned by lift-off technique, resulting a device channel width/length (W/L) of 100/20 $\mu \mathrm{m}$. Finally, after deposition of the Ti/Au back-gate contact metal, the a-IGZO TFTs are annealed in air at $300^{\circ} \mathrm{C}$ for $1 \mathrm{~h}$.

The measurement procedures are designed as follows: before illumination treatment, the a-IGZO TFT is firstly stressed with a positive gate voltage of $20 \mathrm{~V}$ for a total time of $5000 \mathrm{~s}$ with both its source and drain electrodes grounded. Then, monochromatic light sorted by a monochromator from the output port of a $500 \mathrm{~W} \mathrm{Xe}$ arc lamp is directed onto the sample surface through an optical fiber. The illumination wavelength gradually varies from $750 \mathrm{~nm}$ to $450 \mathrm{~nm}$ with a step spacing of $5 \mathrm{~nm}$. At each selected wavelength, the device under test is illuminated for $2 \mathrm{~min}$, and its transfer characteristics are measured right away without turning off the monochromatic light, which is followed by an additional illumination/measurement cycle.

Transfer curves of the fresh and the stressed a-IGZO TFT are shown in Fig. 1. A positive $\Delta \mathrm{V}_{\text {th }}$ of $\sim 6.0 \mathrm{~V}$ is observed after the positive gate bias stress, which should be caused by field-induced electron trapping at the channel/gate dielectric interface. ${ }^{8}$ Meanwhile, based on simple fitting and calculation, there is no apparent change $(<10 \%)$ for SS as well as $\mu_{\mathrm{FE}}$ of the stressed TFT. This result agrees with past reports that moderate bias stress would not considerably 


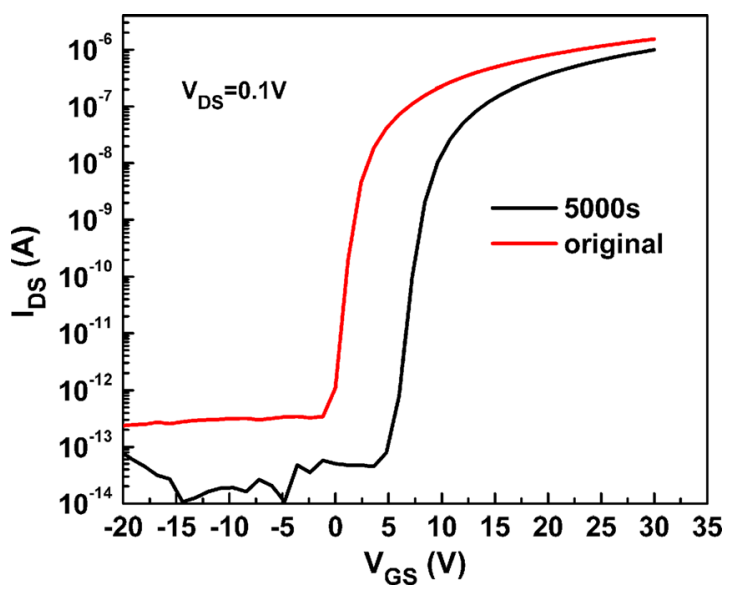

FIG. 1. The transfer curves of the a-IGZO TFT before and after the positive gate bias stress.

generate additional trap states within a-IGZO TFTs. ${ }^{9}$ Therefore, the electrical instability behaviors to be discussed later are mostly caused by light illumination.

When the a-IGZO TFT is exposed to monochromatic light, the device exhibits a series of electrical instabilities. Figures 2(a) and 2(b) show the transfer curves of the stressed a-IGZO TFT after light illumination at various wavelengths in linear scale and logarithmic scale, respectively. First, an overall negative back-shift of the transfer curves is observed as the illumination wavelength decreases from $750 \mathrm{~nm}$ to $450 \mathrm{~nm}$. This negative shift should be caused by photoexcitation of trapped electrons at the channel/dielectric interface. Since electrons trapped at deeper interface states have
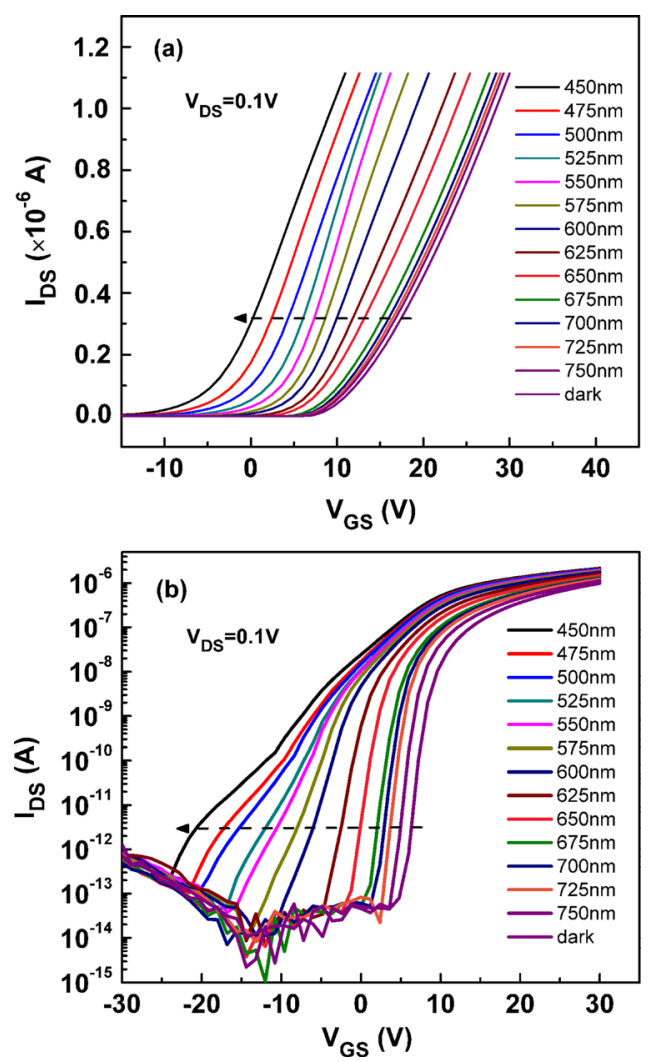

FIG. 2. Transfer characteristics of the stressed a-IGZO TFT after various monochromatic light illumination in linear scale (a) and in logarithmic scale (b). to be released by photons with higher energy, the corresponding $\Delta \mathrm{V}_{\mathrm{th}}$ as a function of illumination wavelength can be used to extract the density profile of interfacial states. ${ }^{10}$ Second, although the transfer curves plotted in logarithmic scale are nearly parallel to each other in long illumination wavelength range, the SS of the TFT exhibits apparent increase at shorter wavelength. Figure 3 shows the SS values as a function of illumination wavelength revealing a turning point at $\sim 625 \mathrm{~nm}$, after which the SS continuously increases. Third, it is found that $\mu_{\mathrm{FE}}$ of the TFT also systemically varies under certain illumination conditions. Here, $\mu_{\mathrm{FE}}$ is calculated by using the following equation: ${ }^{11}$

$$
\mu_{F E}=\frac{L \mathrm{~g}_{m-\max }}{W C_{i} V_{D S}}
$$

where $\mathrm{C}_{\mathrm{i}}$ and $\mathrm{g}_{\mathrm{m}-\max }$ are gate capacitance per unit area and maximum transconductance, respectively. The $\mathrm{g}_{\mathrm{m} \text {-max }}$ is determined by differentiation of the linear-scale transfer curves in Fig. 2(a). As shown in Fig. 3, $\mu_{\mathrm{FE}}$ with an initial value of $\sim 6.7 \mathrm{~cm}^{2} /$ Vs shows fairly small change when the illumination photon energy is below $1.9 \mathrm{eV}$, which is followed by a rapid increase in the photon energy range of $\sim 2.0-2.3 \mathrm{eV}$. After reaching a peak value of $\sim 11.1 \mathrm{~cm}^{2} / \mathrm{Vs}, \mu_{\mathrm{FE}}$ continuously decreases for even larger photon energy.

Comparatively, the electrical instability behaviors of an initial device without undergoing positive-gate-bias stress are also characterized, which exhibit similar changes under monochromatic light illumination. It means that the electrical instability behaviors observed here are intrinsic for a-IGZO TFTs. As shown in Fig. 4 and its inset, besides the same variation trends of SS and $\mu_{\mathrm{FE}}$, a negative shift of $\mathrm{V}_{\mathrm{th}}$ is also observed for an initial device after light illumination. It means that even under equilibrium condition some deep interfacial traps within the a-IGZO TFT are already filled by free electrons. The only difference is that the amount of its illumination-induced $\mathrm{V}_{\text {th }}$ shift is smaller and less apparent than that of the stressed device, which is because that there are less trapped electrons within an initial device.

Our analysis for the above observations focuses on the evolution of SS and $\mu_{\mathrm{FE}}$. It is commonly accepted that the change of SS is mainly depended on the variation of total trap density $\left(\mathrm{N}_{\mathrm{t}}\right)$ within the device active region, which is described by the following equation: ${ }^{12}$

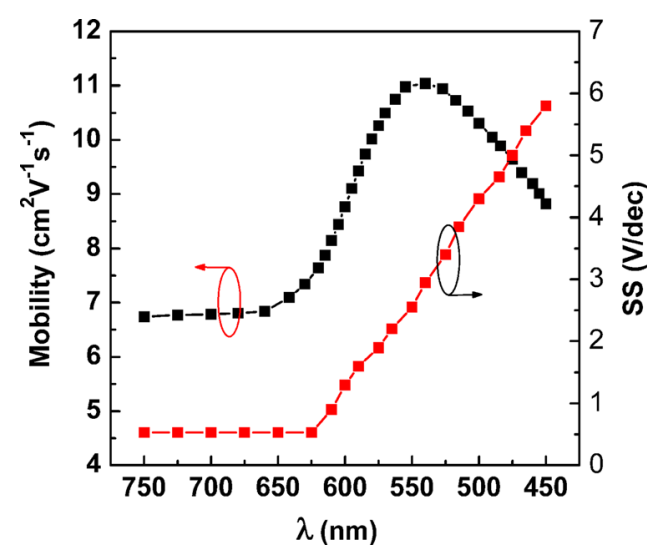

FIG. 3. The $\mu_{\mathrm{FE}}$ and SS of the stressed a-IGZO TFT as a function of light illumination wavelength. 


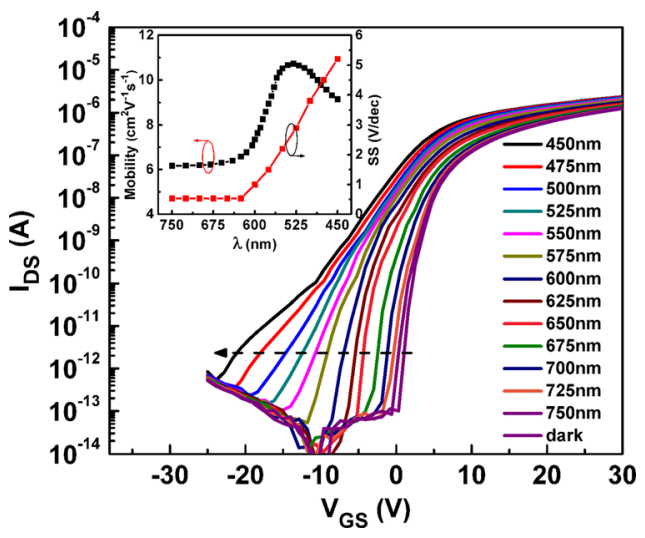

FIG. 4. Transfer characteristics of an initial device after various monochromatic light illumination. The inset shows the $\mu_{\mathrm{FE}}$ and SS of the initial device as a function of illumination wavelength.

$$
\Delta S S=\frac{\Delta N_{t} \ln (10) k T}{C_{i}}
$$

where $k$ is Boltzmann's constant and $T$ is absolute temperature. Thus, the degradation of SS under light illumination of $\lambda \leq 625 \mathrm{~nm}$ can be attributed to extra trap state generation. It has been reported that in oxide-based semiconductors, like aIGZO, defect-induced sub-gap states are commonly originated from oxygen vacancies. ${ }^{13-16}$ In particular, based on theoretical calculation and experimental verifications, neutral oxygen vacancies $\left(\mathrm{V}_{\mathrm{o}}\right)$ would form fully occupied states above valence band maximum (VBM) with an energy width up to $\sim 1.5 \mathrm{eV} .^{6,14}$ Furthermore, the activation energy $\left(\mathrm{E}_{\mathrm{a}}\right)$ needed for the transition of $\mathrm{V}_{\mathrm{o}}$ to singly ionized oxygen vacancies $\left(\mathrm{V}_{\mathrm{o}}^{+}\right)$and double ionized oxygen vacancies $\left(\mathrm{V}_{\mathrm{o}}{ }^{2+}\right)$ were reported to be $\sim 2.0 \mathrm{eV}$ and $\sim 2.3 \mathrm{eV}$, repectively. ${ }^{14,16}$ When photo-induced transition from $\mathrm{V}_{\mathrm{o}}$ to $\mathrm{V}_{\mathrm{o}}{ }^{+}$ occurs, the neighboring metal atoms would undergo small outward relaxation, forming unoccupied defect states near the mid-gap. When photo-induced transition from $V_{o}$ to $\mathrm{V}_{\mathrm{o}}{ }^{2+}$ occurs, the larger outward lattice relaxation in the vicinity of $\mathrm{V}_{\mathrm{o}}{ }^{2+}$ would create a defect level near the bottom of conduction band. ${ }^{14,16,17}$ Here, it is important to note that in our devices, the significant SS shift is observed when the light illumination wavelength $\lambda \leq 625 \mathrm{~nm}(\sim 2.0 \mathrm{eV})$, which agrees well with the activation energy of $\mathrm{V}_{\mathrm{o}}$ to $\mathrm{V}_{\mathrm{o}}{ }^{+}$. Therefore, the defect levels of $\mathrm{V}_{\mathrm{o}}^{+}$and $\mathrm{V}_{\mathrm{o}}{ }^{2+}$ induced by high energy photons should be reason for the increase of total trap density. This proposed SS degradation mechanism is illustrated in Fig. 5.

The above physical picture can also explain the variation of $\mu_{\mathrm{FE}}$ as a function of incident photon energy. When photo-induced transition from $\mathrm{V}_{\mathrm{o}}$ to $\mathrm{V}_{\mathrm{o}}{ }^{+}$occurs, photoexcited electrons from the high-density occupied $V_{o}$ states near valence band maximum would increase average carrier density within the conductive channel, which would then enhance the percolation conduction through distributed potential barriers around the conduction band edge. ${ }^{18,19}$ This effect could explain the increase of $\mu_{\mathrm{FE}}$ in the photon energy range of $\sim 1.9-2.3 \mathrm{eV}$. Although $\mathrm{V}_{\mathrm{o}}{ }^{+}$-related trap states would form around the mid-gap simultaneously, compared with band-tail states near conduction band, these mid-gap states have less influence on channel mobility. When photon

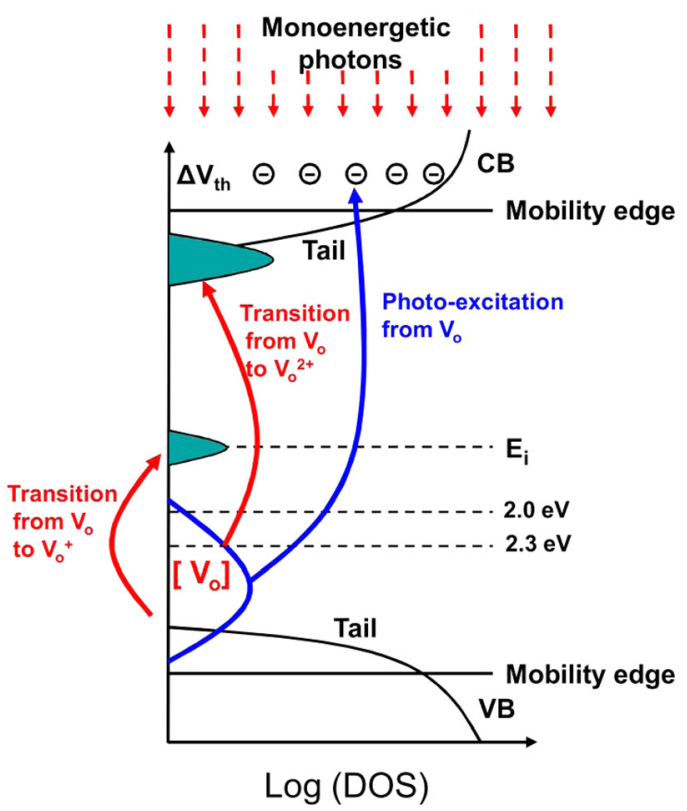

FIG. 5. Schematic illustration of the proposed trap generation process induced by high energy photons.

energy is higher than $2.3 \mathrm{eV}$, transition from $\mathrm{V}_{\mathrm{o}}$ to $\mathrm{V}_{\mathrm{o}}{ }^{2+}$ occurs, which would generate $\mathrm{V}_{\mathrm{o}}{ }^{2+}$-related defect states near the conduction band edge. Since band-tail states are strong scattering centers, $\mu_{\mathrm{FE}}$ would stop increasing and instead start to decrease in spite of the continuously increased channel carrier density. ${ }^{20,21}$

In addition, when $\mu_{\mathrm{FE}}$ is being calculated from the transfer curves at different illumination wavelength, it is found that $\mathrm{g}_{\mathrm{m}-\max }$ occurs at different $\mathrm{V}_{\mathrm{g}}$, which is due to the carrier-detrapping-related $\mathrm{V}_{\text {th }}$ shift. Thus, the factor of $\mathrm{V}_{\mathrm{g}}$ dependence shift in mobility and the variation of its differential on $\mathrm{V}_{\mathrm{g}}$ might also play a role for the anomalous $\mu_{\mathrm{FE}}$ variation as a function of illumination wavelength, which was originally purposed in the reliability study of poly-Si TFTs. ${ }^{22}$

In summary, the effect of monochromatic light illumination on electrical instability behaviors of a-IGZO TFTs is studied in this work. Besides the widely reported $\mathrm{V}_{\mathrm{th}}$ shift, SS and $\mu_{\mathrm{FE}}$ of the TFT are also found to exhibit systemic change at short illumination wavelength range $(\lambda \leq 625 \mathrm{~nm})$. A physical model based on generation of $\mathrm{V}_{\mathrm{o}}{ }^{+}$and $\mathrm{V}_{\mathrm{o}}{ }^{2+}$ related defect states within the a-IGZO active channel by high energy photons is proposed to explain the evolution of device transport properties. In addition, our suggested defect-generation model should be also applicable to an initial device without undergoing positive-gate-bias stress, which is found to exhibit similar SS and $\mu_{\mathrm{FE}}$ evolution behaviors under monochromatic light illumination.

This work was supported by the State Key Program for Basic Research of China under Grant Nos. 2010CB327504, 2011CB922100, 2011CB301900; the National Natural Science Foundation of China under Grant Nos. 60825401, 60936004, 11104130, BK2011556, and BK2011050.

${ }^{1}$ K. Nomura, H. Ohta, A. Takagi, T. Kamiya, M. Hirano, and H. Hosono, Nature (London) 432, 488 (2004). 
${ }^{2}$ H. Yabuta, M. Sano, K. Abe, T. Aiba, T. Den, H. Kumomi, K. Nomura, T. Kamiya, and H. Hosono, Appl. Phys. Lett. 89, 112123 (2006).

${ }^{3}$ K. Nomura, T. Kamiya, M. Hirano, and H. Hosono, Appl. Phys. Lett. 95, 013502 (2009).

${ }^{4}$ B. Kim, E. Chong, D. H. Kim, Y. W. Jeon, D. H. Kim, and S. Y. Lee, Appl. Phys. Lett. 99, 062108 (2011).

${ }^{5}$ S. W. Tsao, T. C. Chang, S. Y. Huang, M. C. Chen, S. C. Chen, C. T. Tsai, Y. J. Kuo, Y. C. Chen, and W. C. Wu, Solid-State Electron. 54, 1497 (2010).

${ }^{6}$ K. Nomura, T. Kamiya, H. Yanagi, E. Ikenaga, K. Yang, K. Kobayashi, M. Hirano, and H. Hosono, Appl. Phys. Lett. 92, 202117 (2008).

${ }^{7}$ T. C. Chen, T. C. Chang, C. T. Tsai, T. Y. Hsieh, S. C. Chen, C. S. Lin, M. C. Hung, C. H. Tu, J. J. Chang, and P. L. Chen, Appl. Phys. Lett. 97, $112104(2010)$

${ }^{8}$ J. Lee, J. S. Park, Y. S. Pyo, D. B. Lee, E. H. Kim, D. Stryakhilev, T. W. Kim, D. U. Jin, and Y. G. Mo, Appl. Phys. Lett. 95, 123502 (2009).

${ }^{9}$ A. Suresh and J. F. Muth, Appl. Phys. Lett. 92, 033502 (2008).

${ }^{10}$ K. Lee, G. Ko, G. H. Lee, G. Han, M. M. Sung, T. W. Ha, J. H. Kim, and S. Im1, Appl. Phys. Lett. 97, 082110 (2010).

${ }^{11}$ M. Kim, J. H. Jeong, H. J. Lee, T. K. Ahn, H. S. Shin, J. S. Park, J. K. Jeong, Y. G. Mo, and H. Dong, Appl. Phys. Lett. 90, 212114 (2007).
${ }^{12}$ Y. Kagan and P. W. E. Andry, Thin Film Transistors (Marcel Dekker, New York, 2003), Chap. 3, p. 87.

${ }^{13}$ T. Kamiya, K. Nomura, M. Hirano, and H. Hosono, Phys. Status Solidi A 206, 860 (2009).

${ }^{14}$ A. Janotti and C. G. Van de Walle, Appl. Phys. Lett. 87, 122102 (2005).

${ }^{15}$ H. Seo, C. J. Park, Y. J. Cho, Y. B. Kim, and D. K. Choi, Appl. Phys. Lett. 96, 232101 (2010)

${ }^{16}$ H. Oh, S. M. Yoon, M. K. Ryu, C. C. Hwang, S. Yang, and S. H. K. Park, Appl. Phys. Lett. 97, 183502 (2010).

${ }^{17}$ B. Ryu, H. K. Noh, E. A. Choi, and K. J. Chang, Appl. Phys. Lett. 97, 022108 (2010).

${ }^{18}$ A. Takagi, K. Nomura, H. Ohta, H. Yanagi, T. Kamiya, M. Hirano, and H. Hosono, Thin Solid Films 486, 38 (2005).

${ }^{19}$ J. M. Lee, I. T. Cho, J. H. Lee, W. S. Cheong, C. S. Hwang, and H. I. Kwon, Appl. Phys. Lett. 94, 222112 (2009).

${ }^{20}$ F. De Angelis, S. Cipolloni, L. Mariucci, and G. Fortunato, Appl. Phys. Lett. 88, 193508 (2006).

${ }^{21}$ S. Lee, K. Ghaffarzadeh, A. Nathan, J. Robertson, S. Jeon, C. Kim, I. H. Song, and U. I. Chung, Appl. Phys. Lett. 98, 203508 (2011).

${ }^{22}$ H. S. Wang, M. X. Wang, Z. Y. Yang, H. Hao, and M. Wong, IEEE Trans. Electron Devices 54, 3276 (2007). 